\title{
Preface the visual computer (Vol 37 issues 12)
}

\author{
Special issue: In memory of Professor Tosiyasu L. Kunii
}

\author{
Nadia Magnenat-Thalmann ${ }^{1}$
}

Accepted: 28 October 2021 / Published online: 6 November 2021

(c) The Author(s), under exclusive licence to Springer-Verlag GmbH Germany, part of Springer Nature 2021

This issue contains a collection of papers written or supervised by researchers who have worked with Professor Kunii and be inspired by him. Professor Kunii, who passed away last November 2020, is the founder of the Visual Computer Journal and the founder of the Computer Graphics Society (CGS). The Visual Computer Journal is the official journal of the Computer Graphics Society (CGS). The Computer Graphics Society (CGS) was officially established in Geneva in 1992 by Professor T.L. Kunii, Nadia Magnenat-Thalmann, Rae Earnshaw, and Daniel Thalmann. CGS is organizing two yearly conferences: Computer Graphics International (CGI) and Computer Animation and Social Agents (CASA).

During CGI2021 held online, a workshop in memory of Professor Kunii has been organized. 17 papers have been invited to contribute to this issue. Selected professors and researchers were invited by Professor Earnshaw to give an homage to Professor Kunii during a Panel. We had also the great pleasure to listen to the testimony of his wife, Dr Hideko Kunii. The papers presentations and the panelists discussions have been recorded. We can find them on YouTube Computer Graphics International 2021 channel.

IEEE Computer Graphics and Applications, through the writing of Professor Michael J. Wozny, has described in detail the career of Professor Kunii (see IEEE CG\&A Issue March/April 2021). This editorial is very exhaustive and we suggest the readers to consult it if they wish to know more details about his full career achievements.

On our side, we would like to say that Professor Kunii has surely and with certainty inspired substantially the academic life of many young researchers by showing them before Mr. Obama, former President of the United States, that yes, we

Special issue: In memory of Professor Tosiyasu L. Kunii.

Nadia Magnenat-Thalmann

thalmann@miralab.ch

1 MIRALab-CUI, University of Geneva, Battelle, Building A, 7, Route de Drize, 1227 Carouge, Geneva, Switzerland can do it, even if one has to go beyond very established networks and institutions. Yes, everybody can go ahead even if a person is at the beginning of her career. We all kept the idea that only hard work pays and to go beyond borders even if the sky is dark. Professor Kunii was very positive as he saw always some blue sky at the horizon. He gave to the academic community an essential and constant message of hope.

We would like, along with all Professors, researchers, former PhD students who have benefited of his great inspiration, to thank him from the heart and to tell him the following: Professor Kunii, you will be with us all the time and we will pursue your efforts further.

Guest Editors: Rae Earnshaw, Nadia Magnenat Thalmann, Xiaoyang Mao, Daniel Thalmann.

In this issue, we present 17 papers:

1. R. Earnshaw, "A new renaissance for creativity in technology and the arts in the context of virtual worlds"

2. Chamin Morikawa et al., "Image and video processing on mobile devices: a survey"

3. Issei Fujishiro et al., "FORSETI; a visual analysis environment for authoring autopsy reports in extended legal medicine mark-up language"

4. Satoshi Nishimura, "Grid-induced bounding volume hierarchy for ray tracing dynamic scenes"

5. Bin Chen, Karol Myskowski et al. "The effect of geometry and illumination on appearance perception of different material categories"

6. Kenji Ohmori, “Formalization of Kublai Khan's globalization using Kunii's incrementally modular abstraction hierarchy"

7. Xiaoyang Mao and Zhenyang Zhu, "Image recoloring for color vision deficiency compensation: a survey"

8. Gauri Tulsulkar, Nadia Magnenat Thalman et al. "Can a humanoid social robot stimulate the interactivity of cognitively impaired elderly? A thorough study based on computer vision methods" 
9. Shigeo Takahashi, "Visual analysis of geospatial multivariate data for investigating radioactive deposition processes"

10. Shin Yoshizawa and Hideo Yokota, "Fast and faithful scale-aware image filters"

11. Zirui Lan, Olga Sourina et al. "Human factors evaluation in VR-based shunting training"

12. Soraia Musse Vinicius Cassol, Daniel Thalmann, "A history of crowd simulation: the past, evolution, and new perspectives"

13. Wenting Dai, Markus Erdt and Alexei Sourin, "Detection and segmentation of image anomalies based on unsupervised defect reparation"
14. Tsukasa Noma et al., "Color2Hatch: conversion of color to hatching for low-cost printing"

15. Taku Komura, "Multi-agent reinforcement learning for character control"

16. Michael Cohen et al., "Directional selectivity in panoramic and pantophonic interfaces: Flashdark, Narrowcasting for Stereoscopic Photospherical Cinemagraphy, Akabeko Ensemble"

17. Wei Li and James Hahn, "Efficient ray casting polygonized isosurface of binary volumes"

Publisher's Note Springer Nature remains neutral with regard to jurisdictional claims in published maps and institutional affiliations. 